\title{
Effects of metformin on middle cerebral artery flow velocity in newly diagnosed type 2 Diabetic patients \\ Hassoun K. Hayder ${ }^{1}$, Ghazal R. Mohammed ${ }^{2}$, Mudhafar MH. Ahmed ${ }^{3}$, Hadi R. Najah ${ }^{3 *}$
}

\begin{abstract}
Type 2 diabetes mellitus is a major cause of cerebrovascular disease. Risk of ischemic stroke is higher in diabetic patients and it is about 2-6 times that in normal population. Atherosclerotic lesions happen early in the disease and patients with type 2 diabetes mellitus. Transcranial Doppler can be used for examination of cerebral arteries and it can detect early atherosclerotic changes. Metformin an old oral anti-diabetic drug but still cornerstone in the treatment guidelines of type 2 diabetes mellitus. It improves endothelial function with preferable cardiovascular effects. Twenty patients were recruited for the study. Transcranial Doppler examination done for all patients before initiation of treatment. Patients received metformin for twelve weeks and they were re-examined with transcranial Doppler. The data of present study showed that a highly significant increase in diastolic velocity and mean flow velocity after treatment with significant reduction in pulsatility index and resistive index. In conclusion, metformin treatment increases cerebral diastolic flow velocity with reduction in vascular resistance in patients with type 2 diabetes.
\end{abstract}

Keywords: Metformin; Transcranial Doppler; Middle cerebral artery (MCA); Type 2 diabetes mellitus

*Corresponding author email: drnajahhadi@ yahoo.com
${ }^{1}$ Department of Neurology, College of Medicine/Kufa University
${ }^{2}$ Department of Pharmacology, College of Medicine/Baghdad University
${ }^{3}$ Department of Pharmacology and Therapeutics, College of Medicine/ Kufa University
Received June13, 2016; accepted August14, 2016; published September14, 2016
Copyright $@ 2016 \mathrm{NH}$. This is article distributed under the terms of the Creative Commons Attribution
License (http://creativecommons.org), which permits unrestricted use, distribution, and reproduction in
any medium, provided the original work is properly cited. (cc) $\mathbf{E Y}$

\section{Introduction}

Stroke is major global health problem. It is considered the 6th cause of death in United States but may be considered the second cause of death in developing countries [1]. There is little data available about risk of stroke in Iraq and even in Middle East area [2]. A study about prevalence of silent stroke in Iraqi Kurdish population was found and it was about $19 \%$ in normal asymptomatic individuals [3]. However, few and old reports from KSA and other Gulf countries recorded annual incidence from 29 to about 60 per 100000 people [4]. The incidence of stroke is increasing in these countries and the risk is expanding, therefore, urgent strategy for prevention and better clinical care of stroke patients is needed [2]. Diabetes mellitus is a well- 
recognized risk factor for cerebrovascular diseases. The association between stroke risk and diabetic patients is well known and many studies concerned with this concept. Previous studies have been found that the risk of stroke and cerebrovascular complication was greater in diabetic patients than those without the disease. The risk was 3 folds greater in patients with type 2 diabetes mellitus than in non-diabetic population. Furthermore, morbidity and mortality that happen with stroke is greater and more severe in diabetic patients than those who are nondiabetic [5]. Also patients with metabolic syndrome and pre-diabetic have one and half fold increase in the risk of stroke than other population [6]. This is due to multiple risk factors like hyperlipidemia, hypertension and insulin resistance which manifested as glucose intolerance.

Early microangiopathic changes are also attributed to cerebrovascular complication and stroke in diabetic patients as macrovascular complications [7]. Transcranial Doppler is useful tool for detection of cerebral vasculopathy. It can detect systolic and diastolic velocities, resistive index and pulsatility index [8-10]. Diastolic flow in cerebral arteries is important because the perfusion of tissues occurs during diastole, therefore it is important to maintain adequate diastolic flow to keep adequate cerebral perfusion and overcome any changes in general circulation [11]. Resistive index and pulsatility index represent vascular resistance [12]. Increase in resistive index means decrease in compliance of artery and atherosclerosis [13]. Increase in pulsatility index means reduction in perfusion of cerebral tissue [14-16]. Metformin is the only member of the biguanide group of oral anti-diabetic drugs available for use today. Metformin is considered the drug of choice for the treatment of patients with type 2 diabetes mellitus. American academy of endocrinology and diabetology guidelines in 2016 mentioned the use of metformin in all stages of diabetes and combined it with other agents in high HbA1c [17, 18]. Metformin is regarded as an oral antidiabetic drug because it lowers blood glucose concentrations in T2D without causing overt hypoglycemia.

Metformin is also frequently described as an insulin sensitizer leading to reduction in insulin resistance and significant reduction of plasma fasting insulin level. The improvement in insulin sensitivity by metformin could be ascribed to its positive effects on insulin receptor expression and tyrosine kinase activity. Metformin is the only biguanide that can be used in clinical practice [18]. In addition to anti-hyperglycemic effect of metformin, it also improves endothelial function. In this regard, it does so by several mechanisms; first: it decreases free fatty acids and triglycerides. Moreover, it inhibits hepatic lipogenesis and increase peripheral fatty acids utilization [19]. Second: metformin mitigates oxidized LDL mediated endothelial injury [20]. Third: it activates SIRT as well as increases its expression in endothelial cells. Previous studies showed that increase SIRT activity and expression is protective 
against endothelial injury and considered as anti-atherosclerotic factor [21]. Fourth: metformin may increase endothelial NO via AMPK activation pathway. Recent studies demonstrated that metformin increases the bioavailability of nitric oxide and eNOS through activation of AMPK pathway [22]. Furthermore, AMPK pathway activation increases the activity of peroxisome proliferator activated receptor gamma which is a transcription factor increases lipid oxidation and utilization in skeletal muscles as well as has important role in regulation of glucose metabolism [23]. Activation of AMPK pathway also reduces endoplasmic reticulum stress which is associated with endothelial dysfunction and accelerated atherosclerotic changes as well as oxidative stress [24]. Fifth mechanism: metformin reduces oxidative stress [25] and finally metformin significantly reduces inflammatory mediators of atherosclerosis like adhesion molecules such as VCAM and ICAM [23].

In previous clinical trial where patients with polycystic ovarian syndrome used metformin, there was significant reduction in vascular oxidative stress, hyperinsulinemia, dyslipidemia and endothelial dysfunction [26]. The net result of above review is that metformin improves endothelial injury and may preserve endothelial function. It has been found that short-duration metformin treatment mitigates arterial stiffness and endothelial dysfunction in young women with polycystic ovary disease [27]. In the United Kingdom Prospective Diabetes Study, metformin was unique among other oral hypoglycemic agents in reduction of cardiovascular morbidity and mortality that is associated with diabetes mellitus [28]. Besides that, metformin reduces risk of retinopathy, neuropathy and other microvascular complications [29]. Metformin also prevent cerebrovascular disease and remodeling in diabetic patient and reduces risk of ischemic stroke as well as it may reverse established diabetes-related vascular structural changes [30]. Our study is conducted to confirm the beneficial effect of early treatment of metformin on cerebral hemodynamics in patients with type 2 diabetes mellitus as examined by transcranial Doppler.

\section{Subjects and methods}

Twenty patients with newly diagnosed type 2 diabetes mellitus were included in our study. The study started from November 2014 and end in October 2016. A verbal consent was taken from each participant. The study was approved by Kufa Medical College Ethical Committee for clinical trials. The diabetic patients were selected from center of diabetes and endocrinology in Al Sadr medical City in Najaf City/Iraq. They were diagnosed with diabetes mellitus type 2 according to World Health Organization definition of diabetes as fasting plasma glucose $\geq 7.0 \mathrm{mmol} / \mathrm{L}$ (126 mg/dL), 2-hour post-load plasma glucose $\geq 11.1 \mathrm{mmol} / \mathrm{L}(200 \mathrm{mg} / \mathrm{dL})$ or HbA1C $\geq 6.5 \%$ [31]. Patients were randomly selected by endocrinologist after diagnosis and all of them have newly diagnosed type 2 diabetes. They 
underwent investigation regarding fasting blood sugar and glycated hemoglobin. Then transcranial Doppler examination and measurements were under taken at TCD department/ Middle Euphrates Neuroscience center/ Al Sadr Medical city/Najaf. Studies were conducted in the morning (9:00 am) after overnight fasting and subjects refrained from products containing caffeine 24-hours before study sessions. The time required for every subject to complete study sessions was approximately 30 minutes. Digital transcranial Doppler with M-mode WAKIe with continuous monitoring and physiological test software (Atysmedical, France) and EZ-Dop DWL, COMPUMEDICS, GmbH, Germany. These instruments are provided with head-band as probe holder for pulse-wave $2 \mathrm{MHz}$ phase array transducer. To study the MCA blood flow velocities, the subject was asked to tilt his head to a side and to breath normal quiet breathing, the MCA was first identified by Doppler probe through trans-temporal window, usually M1 segment. For identification of middle cerebral artery, spectral wave form window was started at $20 \mathrm{~mm}$ and then decreased gradually to $12 \mathrm{~mm}$ to get rid of ultrasound noise and obtain accurate measure. The depth of Doppler beam that was adjusted at $45-55 \mathrm{~mm}$, utilizing a trans-temporal window above zygomatic arch in the preauricular area. To obtain MCA spectral wave form, probe is directed backward and slightly upward. The pulse wave Doppler sample volume was adjusted to maximum level in the device and the power $75 \mathrm{MW}$ for identification and localization of middle cerebral artery spectral waveform [32, 33]. To recognize the waveform of middle cerebral artery, the waves are above zero line in spectral wave display screen. After localization of middle cerebral artery waveform and getting the optimal signal of middle cerebral artery. The means of peak systolic velocity, diastolic velocity, resistive index and pulsatility index of 10 cardiac cycles were automatically recorded by specific TCD software of the device. Then, patients were treated with metformin (Glucophage) 1000mg daily for twelve weeks. After twelve weeks, glycated hemoglobin level with transcranial Doppler examination were repeated.Version 20 SPSS statistical program was used for analysis of data. Paired t-test was used for comparison between means of before and after treatment analysis for the same patients.

\section{Results}

Mean age of patients was $44.55 \pm 1.18$ years. Patients consist of seven women with a ratio of $35 \%$ and 13 men with a ratio of $65 \%$. Metformin group also showed a significant decrease in FBS from $159.25 \pm 6.24 \mathrm{mg} / \mathrm{dL}$ to $129.1 \pm 5.37 \mathrm{mg} / \mathrm{dL}$ after twelve weeks of treatment with metformin $1 \mathrm{~g} /$ day $(\mathrm{P}$ $<0.000)$. For metformin group HbA1c decreased from $7.76 \pm 0.26 \%$ to $6.86 \pm 0.16 \%$ ( $\mathrm{P}<0.000)$. There was no statistical difference in peak systolic velocity between baseline reading $(71.35 \pm 1.84 \mathrm{~cm} / \mathrm{sec})$ and post-treatment one $(72.1 \pm 1.1 \mathrm{~cm} / \mathrm{sec}) \quad(\mathrm{P}=0.517)$. On the hand, there was statistical significant increase in diastolic velocity 
and mean flow velocity from $(25.35 \pm 0.96 \mathrm{~cm} / \mathrm{sec}, 40.68 \pm 1.02 \mathrm{~cm} / \mathrm{sec})$ to $(28.6 \pm 0.5 \mathrm{~cm} / \mathrm{sec}, 43.11 \pm 0.51 \mathrm{~cm} / \mathrm{sec})$ respectively and $\mathrm{p}$ values as follow ( $\mathrm{P}<0.000$ and 0.001). Metformin group showed significant decrease in pulsatility index and resistive index after treatment. Pulsatility index deceased from $1.14 \pm 0.04$ to $1.01 \pm 0.025$ $(\mathrm{P}=0.001)$ and resistive index also decreased from $0.64 \pm 0.0134$ to $0.61 \pm 0.009(\mathrm{P}=0.007)$.

Table 1.

Effect of metformin on glycemic control and Doppler indices

\begin{tabular}{|l|l|l|l|}
\hline \multirow{2}{*}{ Parameter } & \multicolumn{3}{|c|}{ Metformin group } \\
\cline { 2 - 5 } & \multicolumn{3}{|c|}{ Mean \pm SEM } \\
\cline { 2 - 5 } & Before & After & P value \\
\hline FBS (mg/dL) & $159.25 \pm 6.24$ & $129.1 \pm 5.37$ & 0.000 (H.S) \\
\hline HbA1c \% & $7.76 \pm 0.26$ & $6.86 \pm 0.16$ & 0.000 (H.S) \\
\hline Peak systolic velocity (cm/sec) & $71.35 \pm 1.84$ & $72.1 \pm 1.1$ & 0.517 (N.S) \\
\hline Diastolic velocity (cm/sec) & $25.35 \pm 0.96$ & $28.6 \pm 0.5$ & 0.000 (H.S) \\
\hline Mean velocity (cm/sec) & $40.68 \pm 1.02$ & $43.11 \pm 0.51$ & 0.001 (S) \\
\hline Pulsatility Index & & $1.01 \pm 0.025$ & 0.001 (S) \\
\hline Resistive Index & $1.14 \pm 0.04$ & $0.61 \pm 0.009$ & 0.007 (S) \\
\hline
\end{tabular}

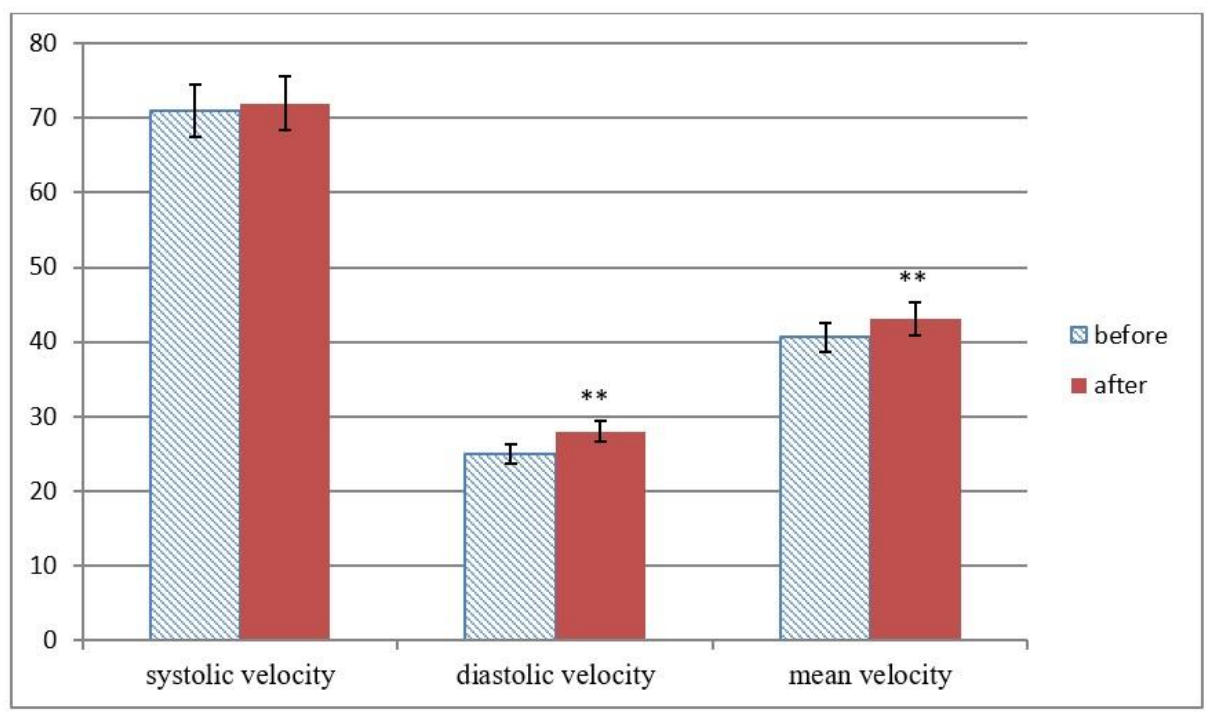

Figure 1.

Bar charts explain the effect of metformin on systolic, diastolic and mean velocities of middle cerebral artery by TCD examination in newly diagnosed patients with type 2 DM. ** means $\mathrm{P}<0.05$. 


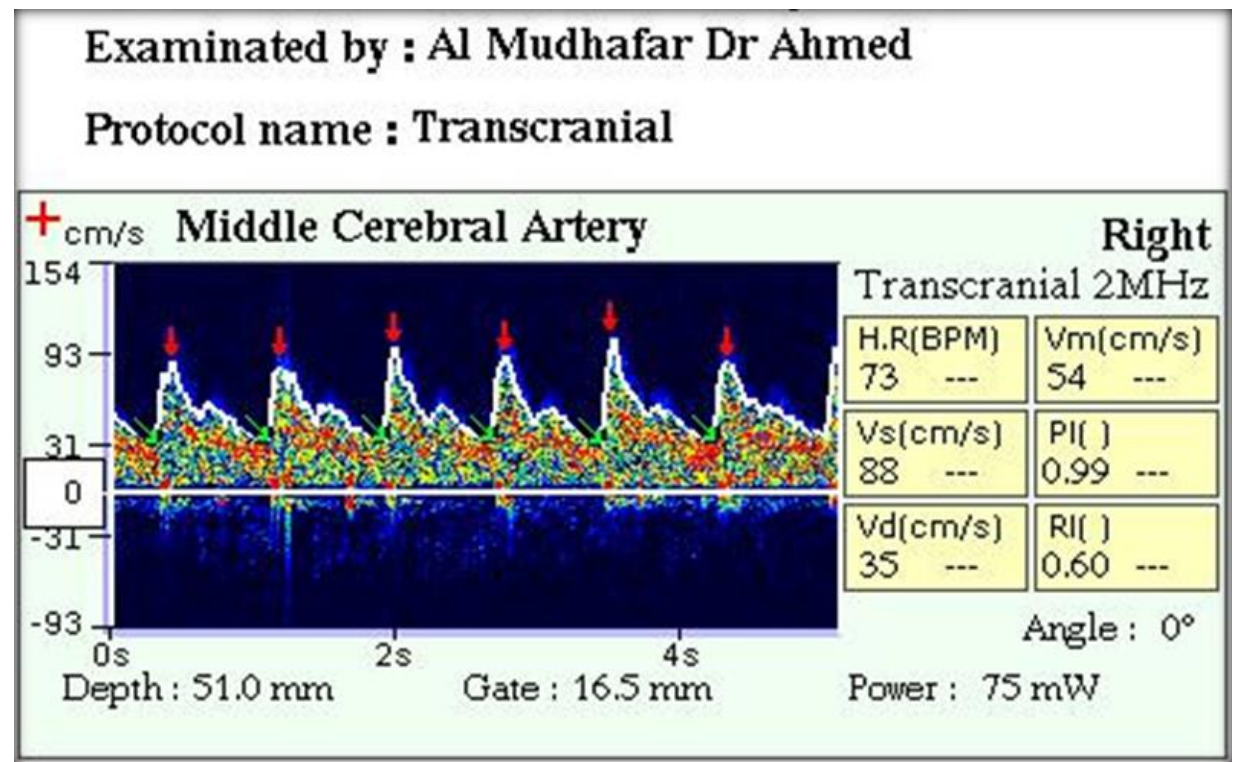

Figure 2.

Spectral wave form of right middle cerebral artery of patient with newly diagnosed type 2 DM before treatment. The yellow arrow referred to diastolic wave.

\section{Examinated by : Al Mudhafar Dr Ahmed}

Protocol name : Transcranial

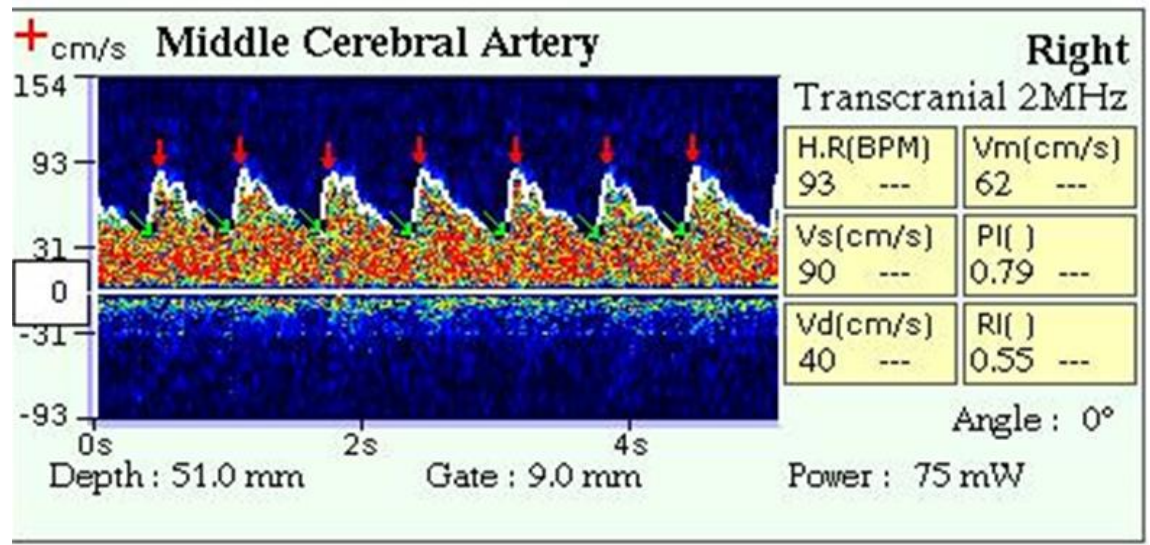

Figure 3.

Spectral waveform of right middle cerebral artery of the same patient above in Figure 2 after treatment with metformin for 12 weeks. The yellow arrow referred to diastolic velocity. There was increase in both systolic and diastolic but the latter is more with decrease in both RI and PI. 


\section{Discussion}

Nowadays, there is clear and sufficient evidence that type 2 diabetes mellitus is associated with accelerated atherosclerosis, therefore an increased risk of stroke and other vascular disease exists. These vascular complications are associated with high morbidity and mortality. From this point, it is of paramount importance to predict any evidence of vasculopathy and manage it earlier [34].

In our study, metformin exerted significant reduction in blood glucose and HbAlc. It is well known that metformin decreases insulin resistance in all tissues especially the liver [35]. It also decreases hepatic output of glucose [36]. Moreover, metformin decreases $\beta$ cells apoptosis and slows diabetes disease progression [37-39]. Many of these effects are mediated through AMP-kinase pathway [22, 23, 30]. Nowadays, metformin use is recommended in the onset of diabetes type 2 [17]. Furthermore, it is highly recommended in pre-diabetic patients and metabolic syndrome [40]. Localized baseline increase in systolic velocity may indicate stenosis of cerebral vessel [41]. In our study, we did not record abnormally high systolic velocity and patients with high systolic velocity that indicate variable degree of stenosis were excluded from our study. On the other hand, diastolic velocity is important as diastolic flow is vital for brain perfusion as cerebral perfusion occurs during diastole $[11,12]$. Patients with impaired diastolic flow have high risk of developing cerebrovascular accident $[13,14]$.
Therefore, improvement in diastolic velocity may protect the patient from ischemic stroke as it keeps adequate blood flow during diastole. Pulsatility index and resistive index may reflect the compliance of the vessel and therefore, high PI and/or RI may indicate increase vascular resistance and increase in arterial stiffness [15, 16]. Previous studies showed that diabetic patients have higher resistive index than normal population and this may increase micro-and macro-vascular complications [32].

Furthermore, it may predict the risk of cerebrovascular disease. It is clear fact that early diagnosis and treatment of vascular complications in diabetic patients is prompt and it is highly recommended. In our study, metformin not only improve diastolic blood flow velocity but it also lower resistive and pulsatility indices. No previous study was found regarding the effect of metformin on cerebrovascular hemodynamics by transcranial Doppler. This finding further ensures the positive cardiovascular effects of metformin. Early use of metformin with regular exercises and dietary control delays the onset of cardiovascular complications of diabetes [42]. In a large randomized clinical trial, metformin decreases the risk of stroke in diabetic patients [43]. Moreover, chronic metformin treatment significantly reduces infarct size in animal model [44]. The mechanism beyond that is chronic metformin therapy down-regulate AMPK in cerebral vessels and decreases lactate accumulation during ischemia [42-45]. However, acute metformin treatment during ischemic stroke may cause 
activation of AMPK which has harmful effects on neurons during ischemia with accumulation of lactate [44].

We concluded, that chronic metformin therapy has beneficial effect on cerebrovascular hemodynamics and may lower the risk of stroke in patients with type 2 diabetes mellitus.

\section{Acknowledgement}

We are grateful for the help of staff member of Middle Euphrates Neuroscience Center/Najaf/Iraq. We are also thankful for great help and support of staff of Center of Diabetes and Endocrine Diseases in Al Sadr Medical City/ Najaf/Iraq.

\section{References}

1. Mozaffarian D, Benjamin J, Go S, et al. Heart disease and stroke statistics-2016 update: a report from the American heart association. Circulation 2015;132:4.

2. Tran J, Mirzaei M, Anderson L, et al. The epidemiology of stroke in the Middle East and North Africa. Journal of the neurological sciences 2010;295:1-2.

3. Al-Shimmery K, Amein H, Al-Tawil G. Prevalence of silent stroke in Kurdistan, Iraq. Neurosciences (Riyadh) 2010;15:16771.

4. Robert A, Zamzami M. Stroke in Saudi Arabia: a review of the recent literature. Pan African Medical Journal 2014;17:14.

5. Martín I, Sevillano C, Segura A, et al. Type 2 diabetes and cardiovascular disease: Have all risk factors the same strength? World Journal of Diabetes 2014;5:444-470.

6. McFarlane I, SicaA, Sowers R. stroke in patients with diabetes and hypertension. The journal of clinical hypertension 2005;7:286294.

7. Zhou H, Zhang X, Lu J. Progress on diabetic cerebrovascular diseases. Bosnian Journal of Basic Medical Sciences 2014; 14:185-190.
8. Widder B. Cerebral vasoreactivity. In: Michael G. H. and Stephen P. M., editor. Cerebrovascular ultrasound. $1^{\text {st }}$ ed. Cambridge: Cambridge University Press; 2001. Pp 324-333.

9. Kassab Y, Majid A, Farooq U, et al. Transcranial Doppler: An Introduction for Primary Care Physicians. J Am Board Fam Med 2007;20:65-71.

10. Sloan A, AlexandrovV, Tegeler H, et al. Assessment: Transcranial Doppler ultrasonography: Report of the Therapeutics and Technology Assessment Subcommittee of the American Academy of Neurology. Neurology 2004;62:1468-81.

11. Guyton C, Hall E. Cerebral Blood Flow, Cerebrospinal fluid, and brain metabolism. In: Arthur C. Guyton and John E. Hall, editors. Textbook of medical physiology; 11th edition: Philadelphia: Elsevier Inc.;2006. pp761-764.

12. McHedlishvili G. Physiological mechanisms controlling cerebral blood flow. Stroke 1980;11:240-48.

13. Mitchell F, Van Buchem A, Sigurdsson S, et al. Arterial stiffness, pressure and flow pulsatility and brain structure and function: the Age, Gene/Environment Susceptibility Reykjavik Study. Brain 2011;134:33983407.

14. Kidwell S, El-Saden S, Livshits Z, et al. Transcranial Doppler pulsatility indices as a measure of diffuse small vessel disease. $J$ Neuroimaging 2001;11:229-235.

15. Wijnhoud D, Koudstaal J, Dippel W. The prognostic value of pulsatility index, flow velocity, and their ratio, measured with TCD ultrasound, in patients with a recent TIA or ischemic stroke. Acta NeurolScand 2011;124:238-44.

16. Bai H, Chen R, Chiu C, et al. Lower blood flow velocity, higher resistance index, and larger diameter of extracranial carotid arteries are associated with ischemic stroke independently of carotid atherosclerosis and cardiovascular risk factors. $J$ Clin Ultrasound 2007;35:322-30.

17. Abrahamson J, Barzilay I, Blonde L, et al. AACE/ACE comprehensive type 2 diabetes management algorithm 2016. EndocrPract 2016;22:84-113. 
18. Strack T. Metformin: a review. Drugs Today (Barc) 2008;44:303-14.

19. Fontbonne A, Charles A, Juhan-Vague I, et al. The effect of Metformin on the metabolic abnormalities associated with upper body fat distribution. Results of the BIGPRO 1 trial. Diabetes Care 1996;19: 920-6.

20. Dong Y, Zhang M, Wang S, et al. Activation of AMP-Activated Protein Kinase Inhibits Oxidized LDL-Triggered Endoplasmic Reticulum Stress In Vivo. Diabetes 2010;59:1386-396.

21. Davis J, Xie Z, Viollet B, et al. Activation of the amp-activated kinase by antidiabetes drug metformin stimulates nitric oxide synthesis in vivo by promoting the association of heat shock protein 90 and endothelial nitric oxide synthase. Diabetes 2006;55:496-505.

22. Lee H, Kim G. AMPK-dependent metabolic regulation by PPAR agonists. PPAR Research 2010;2010:549101.

23. Hwang L, Jeong $\mathrm{T}$, Li X, et al. Inhibitory cross-talk between the AMPK and ERK pathways mediates endoplasmic reticulum stress-induced insulin resistance in skeletal muscle. British Journal of Pharmacology 2013;169:69-81.

24. De la Rosa C, Vrenken E, Buist-Homan M, et al. Metformin protects primary rat hepatocytes against oxidative stress-induced apoptosis. Pharmacology Research \& Perspectives 2015;3:e0125.

25. Viollet B, Guigas B, Sanz Garcia N, et al. Cellular and molecular mechanisms of metformin: an overview. Clinical Science (London, England: 1979) 2012;122:253270.

26. Kocer D, Bayram F, Diri H. The effects of metformin on endothelial dysfunction, lipid metabolism and oxidative stress in women with polycystic ovary syndrome. Gynecol Endocrinol 2014;30:367-71.

27. Agarwal N, Rice S, Bolusani H, et al. Metformin Reduces Arterial Stiffness and Improves Endothelial Function in Young Women with Polycystic Ovary Syndrome: A Randomized, Placebo-Controlled, Crossover Trial. J ClinEndocrinolMetab 2010;95:722-30.
28. King P, Peacock I, Donnelly R. The UK Prospective Diabetes Study (UKPDS): clinical and therapeutic implications for type 2 diabetes. British Journal of Clinical Pharmacology 1999;48:643-48.

29. Bailey J. Metformin: effects on micro and macrovascular complications in type 2 diabetes. Cardiovasc Drugs Ther 2008;22: 215-24

30. Jia J, Cheng J, Ni J, et al. Neuropharmacological actions of metformin in stroke. Current Neuropharmacology 2015;13:38994.

31. American Diabetes Association. Classification and Diagnosis of Diabetes. Diabetes Care 2016;39:S13-S22.

32. Amran G, Zwain A, Hadi N, Al-Mudhaffer A. Autonomic cerebral vascular response to sildenafil in diabetic patient. Diabetology\& Metabolic Syndrome 2012;4:2.

33.Zwain A. A Study of Cerebral Vasoreactivity: Middle Cerebral Artery (MCA) Versus Ophthalmic Artery (OPA). Karbala J. Med 2009;2:604-15.

34. Marshall M, Flyvbjerg A. Prevention and early detection of vascular complications of diabetes. BMJ: British Medical Journal 2006;333:475-80.

35. Pryor R, Cabreiro F. Repurposing metformin: an old drug with new tricks in its binding pockets. Biochemical Journal 2015;471:307-22.

36. Gong L, Goswami S, Giacomini M, et al. Metformin pathways: pharmacokinetics and pharmacodynamics. Pharmacogenetics and genomics 2012;22:820-27.

37. Marchetti P, Del Guerra S, Marselli L, et al. Pancreatic islets from type 2 diabetic patients have functional defects and increased apoptosis that are ameliorated by metformin. Journal of Clinical Endocrinology and Metabolism 2004;89: 5535-41.

38. Forouzandeh F, Salazar G, Patrushev N, et al. Metformin Beyond Diabetes: Pleiotropic Benefits of Metformin in Attenuation of Atherosclerosis. Journal of the American Heart Association: Cardiovascular and Cerebrovascular Disease 2014;3:e01202.

39. Vitale C, Mercuro G, Cornoldi A, et al. Metformin improves endothelial function in 


\section{American Journal of BioMedicine}

patients with metabolic syndrome. Journal of Internal Medicine 2005;258:250-56.

40. Tankova T. Current indications for metformin therapy. Rom J Intern Med 2003;41(3):215-25.

41. Sloan A, Alexandrov V, Tegeler $\mathrm{H}$, et al. Assessment: transcranial doppler ultrasonography: report of the therapeutics and technology assessment subcommittee of the American Academy of Neurology. Neurology 2004;62:1468-81.

42. Lamanna C, Monami M, Marchionni N, et al. Effect of metformin on cardiovascular events and mortality: a meta-analysis of randomized clinical trials. Diabetes ObesMetab 2011;13:221-28.
43. Cheng Y, Leu B, Chen J, et al. Metformininclusive therapy reduces the risk of stroke in patients with diabetes: a 4-year follow-up study. J Stroke Cerebrovasc Dis 2014; 23:e99-105.

44. Li J, Benashski E, Venna V, et al. Effects of Metformin in Experimental Stroke. Stroke; a journal of cerebral circulation 2010;41:2645-52.

45. Ashabi G, Khalaj L, Khodagholi F, et al. Pre-treatment with metformin activates Nrf2 antioxidant pathways and inhibits inflammatory responses through induction of AMPK after transient global cerebral ischemia. Metab. Brain Dis 2015;30:74754.

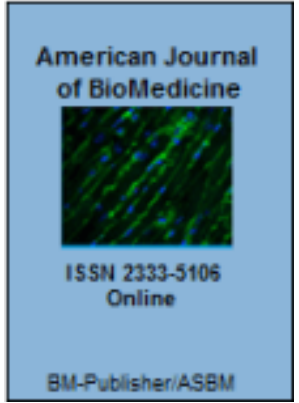

